\title{
Dissociable Neural Substrates for Agentic versus Conceptual Representations of Self
}

\section{Citation}

Powell, Lindsey J., C. Neil Macrae, Jasmin Cloutier, Janet Metcalfe, and Jason P. Mitchell. Forthcoming. Dissociable neural substrates for agentic versus conceptual representations of self. Journal of Cognitive Neuroscience 22.

\section{Published Version}

doi:10.1162/jocn.2009.21368

\section{Permanent link}

http://nrs.harvard.edu/urn-3:HUL.InstRepos:3638679

\section{Terms of Use}

This article was downloaded from Harvard University's DASH repository, and is made available under the terms and conditions applicable to Other Posted Material, as set forth at http:// nrs.harvard.edu/urn-3:HUL.InstRepos:dash.current.terms-of-use\#LAA

\section{Share Your Story}

The Harvard community has made this article openly available.

Please share how this access benefits you. Submit a story.

\section{Accessibility}




\title{
Dissociable Neural Substrates for Agentic versus Conceptual Representations of Self
}

\author{
Lindsey J. Powell ${ }^{1}$, C. Neil Macrae ${ }^{2}$, Jasmin Cloutier ${ }^{3}$, Janet Metcalfe ${ }^{4}$, \\ and Jason P. Mitchell ${ }^{1}$
}

\begin{abstract}
Although humans generally experience a coherent sense of selfhood, we can, nevertheless, articulate different aspects of self. Recent research has demonstrated that one such aspect of self-conceptual knowledge of one's own personality traits - is subserved by ventromedial prefrontal cortex (vMPFC). Here, we examined whether an alternative aspect of "self"being an agent who acts to achieve one's own goals-relies on cognitive processes that overlap with or diverge from conceptual operationalizations of selfhood. While undergoing fMRI, participants completed tasks of both conceptual selfreference, in which they judged their own or another person's personality traits, and agentic self-reference, in which they
\end{abstract}

freely chose an object or watched passively as one was chosen. The agentic task failed to modulate vMPFC, despite producing the same memory enhancement frequently observed during conceptual self-referential processing (the "self-reference" effect). Instead, agentic self-reference was associated with activation of the intraparietal sulcus (IPS), a region previously implicated in planning and executing actions. Experiment 2 further demonstrated that IPS activity correlated with later memory performance for the agentic, but not conceptual, task. These results support views of the "self" as a collection of distinct mental operations distributed throughout the brain, rather than a unitary cognitive system.

\section{INTRODUCTION}

One of the most puzzling-yet unavoidable-features of the human mind is our introspective experience of being a coherent "self." Except in rare instances of pathology, we experience ourselves as having stable, idiosyncratic personality traits and a single, introspective awareness; we view ourselves as the authors of our own, freely willed actions and as the protagonists in a unique autobiographical narrative; and we demarcate ourselves as individuals who, although distinct from other people, are nevertheless defined by our roles, obligations, and personal rights within a larger society. Breakdowns in one or more of these aspects of selfhood have been implicated in a variety of psychiatric and neuropsychological disorders, ranging from schizophrenia (Frith, 2005; Stirling, Hellewell, \& Quraishi, 1998), to alien hand syndrome (Doody \& Jankovic, 1992; Feinberg, Schindler, Flanagan, \& Haber, 1992), to loss of one's personal identity (Klein, Rozendal, \& Cosmides, 2002; Klein, Loftus, \& Kihlstrom, 1996).

Although attempts to understand the nature of this complex, multifaceted experience of human selfhood have historically been the purview of philosophers, the development of empirical approaches to the self has re-

\footnotetext{
${ }^{1}$ Harvard University, Cambridge, MA, ${ }^{2}$ University of Aberdeen, Aberdeen, Scotland, ${ }^{3}$ MIT, Cambridge, MA, ${ }^{4}$ Columbia University, New York, NY
}

cently emerged as a central enterprise across the cognitive sciences. Much of this work has built on observations, dating back to the 1970s, that processing information in a self-referential manner leads to unique patterns of later behavioral performance. For example, perceivers better remember words that they previously encoded with reference to themselves ("does the word curious describe you?") than to another person, a phenomenon known as the "self-reference effect" (Symons \& Johnson, 1997; Bowers \& Gilligan, 1979; Markus, 1977; Rogers, Kuiper, \& Kirker, 1977). Similarly, the engagement of distinct cognitive processes for self-referential thought is likewise thought to underlie such diverse phenomena as the tendency to overvalue items that belong to oneself (the "the endowment effect"; Kahneman, Knetsch, \& Thaler, 1990); differences in the explanations given for one's own behavior relative to the behavior of others (the "fundamental attribution error"; Jones \& Nisbett, 1971); and the assignment of different probabilities to chance outcomes when perceivers themselves participate in the events, for example, by choosing their own lottery numbers or rolling dice (Langer, 1975, 1977).

Inspired by these observations, researchers have recently brought the techniques of cognitive neuroscience to bear on a specific question about the self: Does self-referential thinking rely on cognitive operations that are distinct from other mental processes? For example, in a series of studies, Heatherton et al. (2006), Macrae, Moran, Heatherton, 
Banfield, and Kelley (2004), and Kelley et al. (2002) examined whether the mnemonic benefits of self-referential processing come about because of differences in the neural systems deployed when thinking about self and others. Providing evidence in favor of such a dissociation, these researchers described a region of ventromedial prefrontal cortex (vMPFC) that is engaged preferentially during introspection about one's own personality traits (compared to those of another person), and, moreover, in which activity during encoding predicts whether self-relevant information will later be remembered or forgotten. To the extent that such neural dissociations reflect corresponding differences in cognitive processing, these data suggest that selfreference does, indeed, engage a unique set of mental operations.

More recent neuroimaging findings have further suggested that a number of different aspects of self all converge on MPFC. For example, in addition to its role in conceptual judgments about one's personality, this region contributes to autobiographical memory (Maguire, 2001), monitoring one's own thoughts (Mason et al., 2007), reporting one's attitudes (Mitchell, 2009b), and the subjective experience of emotion (Phan, Wager, Taylor, \& Liberzon, 2002). Indeed, such findings have prompted a number of researchers to posit a general role for MPFC (and related midline cortical regions) in subserving "the self," broadly construed (Schneider et al., 2008; Uddin, Iacoboni, Lange, \& Keenan, 2007; Northoff \& Bermpohl, 2004; cf. Gillihan \& Farah, 2005).

Recent cognitive work has likewise suggested the unity of self, by demonstrating that different kinds of selfreferential processing can produce common behavioral effects. For example, extending the classic self-reference effect in memory, Cloutier and Macrae (2008) demonstrated enhanced recall for self-relevant information with a task in which participants alternated between freely acting over an item (choosing numbered slips of paper from a bowl) and passively watching another person perform the same task. That is, situations in which participants experienced agency - the sense of acting deliberately and freely on an object of one's choice-were associated with the same augmented memory performance as situations, studied since the 1970s, in which they introspect conceptually about their personality traits and dispositions.

However, commentators since William James (1890) have highlighted the complex and paradoxical nature of the self. Although we typically experience "self" as a singular entity, we can, nevertheless, conceive of and articulate different aspects of selfhood (also see Boyer, Robbins, \& Jack, 2005; Gallagher, 2000; Humphrey, 2000; Neisser, 1988). This recognition of the multifaceted nature of our first-person experience has prompted a critical question about the cognitive architecture underlying the various aspects of self: Does our overall sense of selfhood emerge from a single, discrete set of mental processes or is the "self" better conceptualized as a confederacy of different cognitive mechanisms, each performing some unique function? In other words, having established that at least some experiences of selfhood rely on cognitive processes distinct from non-self-referential mentation, researchers have begun to ask a second-level question about dissociations among different aspects of self: Do the various experiences of self draw on a single set of processes that are specialized for representing self-relevant information, or do different aspects of self draw on distinct cognitive mechanisms?

Indeed, there are several reasons to suspect that agentic aspects of self will differ in important respects from the more "conceptual" and autobiographic aspects of self that have been linked to MPFC. Recently, researchers have implicated MPFC in tasks that require the suspension of current, externally directed processing in favor of internally focused mentation that is detached from the immediate perceptual environment (for review, see Mitchell, 2009a; Buckner \& Carroll, 2007). Consistent with these observations, the aspects of self known to rely on MPFC involve exactly this kind of introspective, abstract, and context-free processing; for example, autobiographical memory requires that one transcend the current situation and mentally simulate the past, whereas thinking about one's personality traits demands integration over a lifetime of relevant episodes in which a particular trait could be expressed. In contrast, the sense of oneself as an agent is inherently tied to the here-and-now. As defined by Wegner (2002), our conscious sense of agency is "experienced when we perform an action-actions feel willed or not, and this feeling of voluntariness or doing a thing 'on purpose' is an indication of conscious will" (p. 3). Successful recognition of oneself as the free author of an action requires attention not only to one's current movements but to the effects of such behavior on the external environment. A sense of agency cannot be achieved through detachment from the immediate perceptual context, but only arises out of the coupling of internal goals with their consequences in the environment.

Hence, despite the empirical consensus that "the self" broadly relies on midline structures such as MPFC, we hypothesized that conceptual and agentic aspects of selfhood will rely on distinct cognitive processing and underlying neural substrates. In the current studies, we specifically asked whether the common mnemonic effects of conceptual and agentic self-reference draw, in turn, on common or distinct cognitive processing. Participants completed a standard task of conceptual self-knowledge, in which they alternately judged how well various trait adjectives describe either themselves or a familiar other. In addition, participants completed a second task designed to manipulate the subjective sense of acting agentically toward a specific object. Following the earlier demonstrations by Cloutier and Macrae (2008), this agentic self-reference task alternately required participants to select an object (computerized cards that "turned over" to reveal a word) or obliged them to watch passively as another player selected one. As in the sizeable number of studies that have examined the neural basis of conceptual self-referential processing, we expected to observe greater activity in vMPFC during 
judgments of one's personality than the personality of another person (Ames, Jenkins, Banaji, \& Mitchell, 2008; Jenkins, Macrae, \& Mitchell, 2008; D’Argembeau et al., 2007; Zhu, Zhang, Fan, \& Han, 2007; Heatherton et al., 2006; Mitchell, Macrae, \& Banaji, 2006; Moran, Macrae, Heatherton, Wyland, \& Kelley, 2006; Lou et al., 2004; Macrae et al., 2004; Schmitz, Kawahara-Baccus, \& Johnson, 2004; Johnson et al., 2002; Kelley et al., 2002; Zysset, Huber, Ferstl, \& von Cramon, 2002). Critically, to the extent that agentic self-referential processing relies on a similar set of cognitive processes, we also expected to observe similar modulation of vMPFC activity associated with items selected by oneself versus those selected by another person on the agentic selfreference task. In contrast, if one's subjective sense of agency relies on different cognitive processing than one's conceptual self-knowledge, we expected to observe a reliable neural dissociation between the regions modulated by self versus other across the two tasks.

\section{METHODS}

\section{Participants}

Participants were right-handed, native English speakers with no history of neurological problems who gave consent in a manner approved by the Human Studies Committee of the Massachusetts General Hospital. Experiment 1 included 13 participants ( 9 women; mean age $=20$ years; range $=18-24$ years $)$. Experiment 2 included 18 participants ( 8 women; mean age $=20$ years; range $=18-31$ years), in addition to two participants who were excluded due to a technical error that prevented data analysis.

\section{Behavioral Procedure}

\section{Agentic Self-reference Task}

During scanning, participants alternately selected items from a set of 52 "cards" or watched as a computerized partner select an item. Cards were depicted as white, numbered rectangles presented on a black background. At the beginning of each trial, participants viewed a prompt reading either "YOU" or "COMPUTER" in the middle of the screen, which indicated whether the participant or the computer was to select a card. This prompt was displayed for 1 sec and was then replaced by four cards displayed horizontally across the screen (Figure 1). During "self" trials, participants selected a card by pressing one of four response buttons with their right hand. Participants were obliged to make their response within a window of $2 \mathrm{sec}$, after which the selected card was "turned over" to reveal a word. If the participant failed to select a card during the 2 -sec response window, the computer randomly picked the card to reveal; such trials were excluded from subsequent analyses (on average, 8\% of trials across both experiments). During "other" trials, participants were asked to press a fifth button with their right thumb, but were informed that this button press would not affect which card

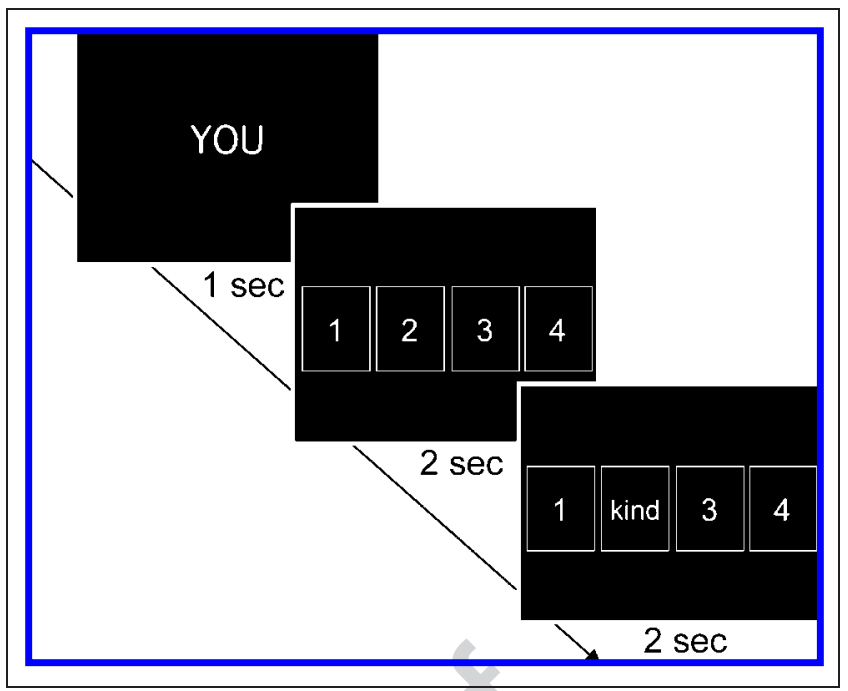

Figure 1. A schematic diagram of trials from the agentic self task. Participants first saw a cue ("YOU" or "COMPUTER") for $1 \mathrm{sec}$ indicating whether they were going to select a card or the computer was. Subsequently, four numbered cards were presented for $2 \mathrm{sec}$, during which time participants selected a card if it was their turn or pressed a dummy key if it was not. Finally, the number on one of the cards disappeared and was replaced by a word for $2 \mathrm{sec}$. Following YOU cues (self trials), the word appeared on the card selected by the participant; following COMPUTER cues (other trials), the word appeared on a randomly selected card.

was selected by the computer. After 2 sec, a randomly selected card was "turned over" by the computer. For both trial types, the word remained onscreen for $2 \mathrm{sec}$, after which the trial ended. In Experiment 1, the set of words comprised a list of positive and negative trait adjectives, whereas in Experiment 2, the stimulus set comprised a list of abstract and concrete nouns. Although the location of the word on "self" trials was determined by participants' selection, the order in which words appeared was predetermined by a randomized computer algorithm.

The numbering of the cards began with " 1 " and proceeded consecutively such that the four cards shown on the first turn were labeled 1 through 4 , the four cards shown on the second turn were labeled 5 through 8, and so on. Once the last four cards had been shown, cards were shown from the beginning of the deck, with the exception that cards that had been selected by the participant or the computer in previous turns were removed from the deck (e.g., if the "2" card had been selected on the first trial, the 14th trial would consist of the cards " 1 " " 3 " "4," and " 5 "). In this way, participants were given the sense that they were choosing a specific card to reveal a specific word. Trials continued to iterate through the deck until all cards had been chosen (i.e., 52 trials). Participants completed two such runs in Experiment 1 (52 self and 52 other trials) and four runs in Experiment 2 (104 self and 104 other trials), each of which was 392 sec in length. To optimize estimation of the event-related fMRI response, trials were intermixed in a pseudorandom order and separated by a variable interstimulus interval (2000-10000 msec; Dale, 
1999), during which participants passively viewed a fixation crosshair.

\section{Conceptual Self-reference Task}

All participants in Experiment 1 and 15 participants in Experiment 2 also completed two runs of the conceptual self-reference task used by Heatherton et al. (2006), Moran et al. (2006), Macrae et al. (2004), and Kelley et al. (2002). On each trial, subjects saw a positive or negative trait adjective and were asked to judge either how well the word described themselves or how well it described then-president George W. Bush by choosing a number from 1 (poor descriptor) to 4 (good descriptor). The target word and a cue indicating either self or Bush were displayed in white text on a black background. Each trial lasted $4 \mathrm{sec}$, and each run consisted of 50 trials (25 of each question type), again intermixed in a pseudorandom order and separated by a variable interstimulus interval (400-8000 msec), for a total run length of $260 \mathrm{sec}$.

\section{Recognition Memory Test}

Immediately after scanning, participants from Experiment 1 completed a surprise recognition memory test for the words they had seen in both the agentic and conceptual self-processing tasks. This memory test comprised a random-ordered presentation of the 104 old words from the agentic self-reference task, the 100 words from the conceptual self-reference task, and 208 novel foils that had not appeared during scanning. For each trial, participants were simply asked to press one of two computer keys to indicate whether the word was "old" or "new." The memory test proceeded in a self-paced manner.

Participants in Experiment 2 completed a similar surprise recognition memory test that comprised a randomly ordered presentation of the 208 words that they had seen during the agentic self-reference task only, along with 208 novel foils. This memory test was administered immediately after the completion of the agentic self-reference task, while participants were still in the scanner (during the collection of the structural data). Participants were given three response options: "old" with high confidence; "old" with low confidence; and "new." Following earlier studies that have examined the neural correlates of successful encoding (Davachi, Mitchell, \& Wagner, 2003; Brewer, Zhao, Desmond, Glover, \& Gabrieli, 1998; Kelley et al., 1998; Wagner et al., 1998), subsequent memory analysis was restricted to items judged "new" or that were remembered with high confidence, so as to ensure that "old" responses truly reflected recollection of the earlier word rather than including serendipitously correct guesses.

\section{Imaging Procedure}

Experiment 1 was conducted using a 1.5-Tesla Siemens Avanto scanner with a standard head coil. A high-resolution
T1-weighted structural scan (MP-RAGE) preceded the four functional runs. Experiment 2 was conducted using a 3.0-Tesla Trio scanner with a standard head coil. A highresolution T1-weighted structural scan (MP-RAGE) was conducted following the four functional runs for the agentic self-reference task and preceding the two functional runs for the conceptual self-reference task. Across both experiments, functional runs used a gradient-echo, echo-planar pulse sequence $(\mathrm{TR}=2 \mathrm{sec}$; $\mathrm{TE}=35 \mathrm{~m}$; $3.75 \times 3.75$ in-plane resolution; 26 axial slices; $5 \mathrm{~mm}$ thick; $1 \mathrm{~mm}$ skip). Each functional run for the agentic selfprocessing task comprised 196 volume acquisitions, whereas each functional run for the conceptual self-processing task comprised 130 volume acquisitions. Stimuli were projected with an Apple MacBook laptop running MATLAB v7.4 and PsyScope for OS X (L. Bonatti, International School of Advanced Studies, Trieste, Italy) onto a screen at the end of the magnet bore that participants viewed by way of a mirror mounted on the head coil. A pillow and foam cushions were placed inside the head coil to minimize head movement.

\section{Data Analysis}

fMRI data were preprocessed and analyzed using SPM2 (Wellcome Department of Cognitive Neurology, London, UK). First, functional data were time-corrected for differences in acquisition time between slices for each wholebrain volume and realigned to correct for head movement. Functional data were then transformed into a standard anatomical space (3-mm isotropic voxels) based on the ICBM 152 brain template (Montreal Neurological Institute). Normalized data were then spatially smoothed $(8 \mathrm{~mm}$ fullwidth-at-half-maximum [FWHM]) using a Gaussian kernel. Statistical analyses were performed using the general linear model, in which trials were modeled using a canonical hemodynamic response function, its temporal derivative, and additional covariates of no interest (a session mean and a linear trend). Comparisons of interest were implemented as linear contrasts using a random-effects model. Regions of interest were identified using a statistical criterion of 25 or more contiguous voxels at a voxelwise threshold of $p<.001$. This cluster size was selected on the basis of a Monte Carlo simulation (S. Slotnick, Boston College) of our brain volume, which found that this cluster extent cutoff provided an experiment-wise threshold of $p<.05$, corrected for multiple comparisons. Additional statistical comparisons between conditions were conducted using ANOVA procedures on the parameter estimates associated with each trial type.

\section{RESULTS}

\section{Experiment 1}

\section{Behavioral Data}

To examine participants' memory for items encountered during the agentic and conceptual self-processing tasks, 
we first calculated a corrected recognition score for each participant as the proportion of previously presented items that were correctly judged to be old (hits) minus the proportion of novel items that were erroneously judged to be old (false alarms). Replicating demonstrations of enhanced memory for items processed self-referentially (Symons \& Johnson, 1997), participants demonstrated better memory on the conceptual self-reference task for trials on which they judged how well an adjective described themselves $(M=0.54, S D=0.15$, range $=0.23$ to 0.77$)$ than another person $(M=0.45, S D=0.18$, range $=0.21$ to 0.77$)$ $[t(12)=3.70, p<.01$, Cohen's $d=1.07$; see Table 1$]$. Likewise, replicating the results of Cloutier and Macrae (2008), participants demonstrated better memory on the agentic self-reference task for trials on which they selected a card $(M=0.21, S D=0.12$, range $=-0.02$ to 0.37$)$ than those on which the computer randomly selected one $(M=0.11$, $S D=0.08$, range $=0.0$ to 0.25$)[t(12)=3.85, p<.003$, $d=1.11]$. On the conceptual task, participants were significantly faster to make judgments during self trials $(M=$ $1739 \mathrm{msec}, S D=137)$ than other trials $(M=1837 \mathrm{msec}$, $S D=193)[t(12)=2.79, p<.05, d=0.81]$. No comparable analysis of response latencies was possible for the agentic task because participants were asked to make a meaningful response only on self trials.

\section{fMRI Data}

Using a whole-brain, random-effects analysis, we identified brain regions that were more active for the comparison of

Table 1. Proportion of "Old" Responses by Trial Type during the Recognition Tests in Experiments 1 and 2

\begin{tabular}{lcc}
\hline \multicolumn{3}{c}{ Experiment 1 } \\
\hline Self & Agentic Task & Conceptual Task \\
Other & $0.43(0.16)$ & $0.77(0.11)$ \\
Novel & $0.34(0.12)$ & $0.68(0.14)$ \\
& $0.22(0.12)$ & $0.23(0.10)$ \\
\hline & & \\
\hline & Experiment 2 & $0.27(0.16)$ \\
Self & High Confidence & $0.30(0.19)$ \\
Other & $0.44(0.14)$ & $0.33(0.21)$ \\
\hline Novel & $0.36(0.17)$ &
\end{tabular}

Values in parentheses represent the standard deviations associated with each trial type. The proportion of "old" responses to novel items is the false alarm rate; corrected recognition scores were calculated by subtracting these values from the corresponding proportion of old responses to words previously presented as either self or other trials. In Experiment 2, participants only completed the recognition memory test for items presented during the agentic task. Responses from this experiment are presented separately for "old" responses accompanied by high and low confidence.
Table 2. Peak Voxel, Brodmann's Area, and Number of Voxels for Brain Regions Obtained from the Random-effects Contrast of Self and Other Trials on the Conceptual Self-reference Task, $p<.05$, Corrected (Experiment 1)

\begin{tabular}{|c|c|c|c|c|c|c|}
\hline Region & $x$ & $y$ & $z$ & $B A$ & Voxels & $t$ \\
\hline \multicolumn{7}{|l|}{ Self $>$ Other } \\
\hline vMPFC & 4 & 32 & 6 & 24 & 658 & 7.56 \\
\hline $\begin{array}{l}\text { Lateral occipito-temporal } \\
\text { sulcus }\end{array}$ & 44 & -58 & -6 & 37 & 475 & 14.10 \\
\hline \multirow[t]{3}{*}{ Cerebellum } & 26 & -44 & -46 & - & 103 & 5.99 \\
\hline & 26 & -44 & -20 & - & 61 & 5.69 \\
\hline & -16 & -46 & -16 & - & 100 & 5.58 \\
\hline Postcentral gyrus & 64 & -30 & 34 & 40 & 76 & 5.80 \\
\hline $\begin{array}{l}\text { L Superior temporal } \\
\text { sulcus }\end{array}$ & -58 & & 18 & 42 & 39 & 4.90 \\
\hline L Inferior frontal gyrus & -60 & 0 & 12 & 6 & 37 & 6.72 \\
\hline $\begin{array}{l}\text { R Superior temporal } \\
\text { sulcus }\end{array}$ & & -34 & 20 & 42 & 36 & 4.97 \\
\hline Occipital cort & -24 & -86 & 26 & 31 & 32 & 4.67 \\
\hline Putamen & -16 & 8 & -8 & - & 34 & 5.16 \\
\hline \multirow[t]{2}{*}{ Cingulate sulcus } & 6 & -32 & 52 & 6 & 30 & 4.86 \\
\hline & -10 & -28 & 44 & 31 & 30 & 4.73 \\
\hline Inferior temporal sulcus & -62 & -8 & -26 & 20 & 39 & 6.60 \\
\hline Orbital gyrus & -48 & 30 & -14 & 47 & 28 & 5.00 \\
\hline
\end{tabular}

$t$ Values reflect the statistical difference between the two conditions, as computed by SPM2. Coordinates refer to the Montreal Neurological Institute stereotaxic space. $\mathrm{BA}=$ Brodmann's area; $\mathrm{vMPFC}=$ ventromedial prefrontal cortex; $\mathrm{R}=$ right; $\mathrm{L}=$ left.

self $>$ other on the conceptual self-reference task. Replicating earlier results, greater BOLD signal was observed for self than other trials in an extensive region of vMPFC (comprising 658 voxels centered at an MNI coordinate of 4, 32, $6)$; additional regions of differential activation included the posterior extent of the inferior temporal gyrus, left inferior frontal gyrus, posterior cingulate, and right motor cortex (Table 2). To examine whether agentic self-referential processing draws on the same processes as those tapped by the conceptual self-reference, we then examined the response in this vMPFC region during the agentic self-reference (card-selection) task. No difference between self-selected and other-selected cards was observed (Figure 1) $[t(12)=$ $0.76, n s, d=0.22]$, suggesting that one's agentic sense of self did not draw on the same cognitive processes as those engaged by conceptual self-reference. That vMPFC response was modulated differently by conceptual and agentic self-reference was confirmed by a significant twoway interaction of Task (conceptual, agentic) $\times$ Chooser (self, other) $[F(1,12)=14.75, p<.003, d=1.11]$. 
Which brain regions were modulated by the agentic selfreference task? To address this question, we used a wholebrain, random-effects analysis to identify the pattern of BOLD response that differentiated between self $>$ other trials on the agentic task. This contrast revealed bilateral anterior intraparietal sulcus (IPS) and right-lateralized regions of the middle and superior frontal gyrus (Table 3), but no differences in medial prefrontal cortex. These four regions were subsequently examined for differences between self and other on the conceptual self-reference task. Specifically, the parameter estimates associated with self (conceptual) and other (conceptual) were extracted from these regions, and ANOVA was used to test for differences between the two trial types. In none of these four regions did we observe any difference between self and other on the conceptual task (all four observed $p$ values were $>$.47). That the response of these regions was modulated differently by conceptual and agentic self-reference was confirmed by significant two-way interactions of Task (conceptual, agentic) $\times$ Chooser (self, other) in all regions identified by the agentic task (all Fs $>6.45$; all $p s<.03$ ). We further observed significant three-way Region $\times$ Trial

Table 3. Peak Voxel, Brodmann's Area, and Number of Voxels for Brain Regions Obtained from the Random-effects Contrast of Self and Other Trials on the Agentic Self-reference Task, $p<.05$, Corrected (Experiment 1)

\begin{tabular}{|c|c|c|c|c|c|c|}
\hline Region & $x$ & $y$ & $z$ & $B A$ & Voxels & $t$ \\
\hline \multicolumn{7}{|l|}{ Self $>$ Other } \\
\hline R Intraparietal sulcus & 44 & -48 & 50 & 40 & 265 & \\
\hline R Middle frontal gyrus & 46 & 28 & 30 & 9 & 117 & 5.92 \\
\hline L Intraparietal sulcus & -48 & -44 & 50 & 40 & 35 & 4.99 \\
\hline R Superior frontal gyrus & 24 & 4 & & 6 & 26 & 5.13 \\
\hline \multicolumn{7}{|l|}{ Other $>$ Self } \\
\hline \multirow[t]{3}{*}{ L Occipital cortex } & -3 & -7 & 24 & 39 & 232 & 9.79 \\
\hline & -10 & -96 & 14 & 18 & 149 & 6.06 \\
\hline & -10 & -80 & 0 & & 28 & 5.66 \\
\hline \multirow[t]{3}{*}{ R Occipital cortex } & 20 & -94 & 10 & 18 & 111 & 7.45 \\
\hline & 20 & -82 & -16 & 18 & 31 & 5.04 \\
\hline & 16 & -88 & 30 & 19 & 47 & 5.03 \\
\hline Superior temporal sulcus & -60 & -16 & -14 & 21 & 172 & 8.47 \\
\hline Supramarginal gyrus & -58 & -58 & 10 & 39 & 162 & 6.07 \\
\hline Putamen & -32 & -4 & 6 & - & 92 & 5.93 \\
\hline Parahippocampal gyrus & -26 & -48 & -8 & 19 & 67 & 8.65 \\
\hline Cerebellum & 2 & -52 & -24 & - & 37 & 5.58 \\
\hline Precentral gyrus & -26 & -40 & 74 & 2 & 29 & 5.68 \\
\hline Insular gyrus & -36 & -6 & -18 & 21 & 25 & 4.73 \\
\hline
\end{tabular}

type $\times$ Task interactions when comparing vMPFC data with each of these regions, all $F$ s $>43.5$, all $p s<10^{-5}$, demonstrating that the pattern of conceptual self $>$ other modulations was qualitatively different in vMPFC than in the four regions isolated by the agentic self-reference task (Figure 2).

\section{Experiment 2}

To test whether brain regions isolated by the agentic self task played a functionally important role in encoding a representation of one's goals and actions, Experiment 2 examined whether the response in such regions during the agentic task correlated with subsequent memory for items selected by participants. Each participant's performance on a test of memory for items in the card selection task was used to retroactively conditionalize encoding items into those that were subsequently remembered versus those that were subsequently forgotten as a function of whether the item had been selected by the participant or by the computer. To the extent that regions such as the IPS play an important, selective role in representing the agentic self, activity in those regions should distinguish between remembered versus forgotten items, but only for those that were initially selected agentically and not for computer-selected items.

\section{Behavioral Data}

Analysis of corrected recognition scores again confirmed that memory performance was better for items that participants self-selected $(M=0.26, S D=0.29$, range $=-0.16$ to 0.82$)$ than those randomly selected by the computer $(M=0.21, S D=0.30$, range $=-0.10$ to 0.81$)[t(17)=$ $2.14, p<.05, d=0.52$; Table 1]. As in Experiment 1 , on the conceptual task, participants were significantly faster to make judgments during self trials $(M=1739 \mathrm{msec}, S D=$ 164) than other trials $(M=1884 \mathrm{msec}, S D=244)[t(15)=$ $3.45, p<.01, d=0.99]$.

\section{fMRI Data}

A whole-brain, random-effects comparison of all self $>$ other trials on the agentic self-reference task replicated the results of Experiment 1, again identifying bilateral regions of the IPS that were associated with a significantly greater response for trials on which participants agentically selected a card versus those on which they passively watched the computer select one. Additional loci were identified in the bilateral superior and middle frontal gyri. In general, the size of the regions identified from this contrast was more extensive than those observed in Experiment 1 , most likely the result of the greater statistical power provided by a larger sample of participants and greater number of trials in Experiment 2, as well as the stronger field strength at which this experiment was conducted (see Table 4). 
Figure 2. Selected brain regions defined from the contrast of self $>$ other, separately for the conceptual (top) and agentic (bottom) self-reference tasks in Experiment 1. Replicating earlier research, vMPFC differentiated between self and other on the conceptual task; however, no difference between trial types was observed in this region for the agentic task. Conversely, bilateral regions of the IPS differentiated between self and other trials on the agentic, but not the conceptual, self-reference task. The right side of each panel displays the parameter estimates associated with each of the four trial types (the bottom displays results from the right IPS). Error bars represent confidence interval for within-subject designs (Loftus \& Masson, 1994).

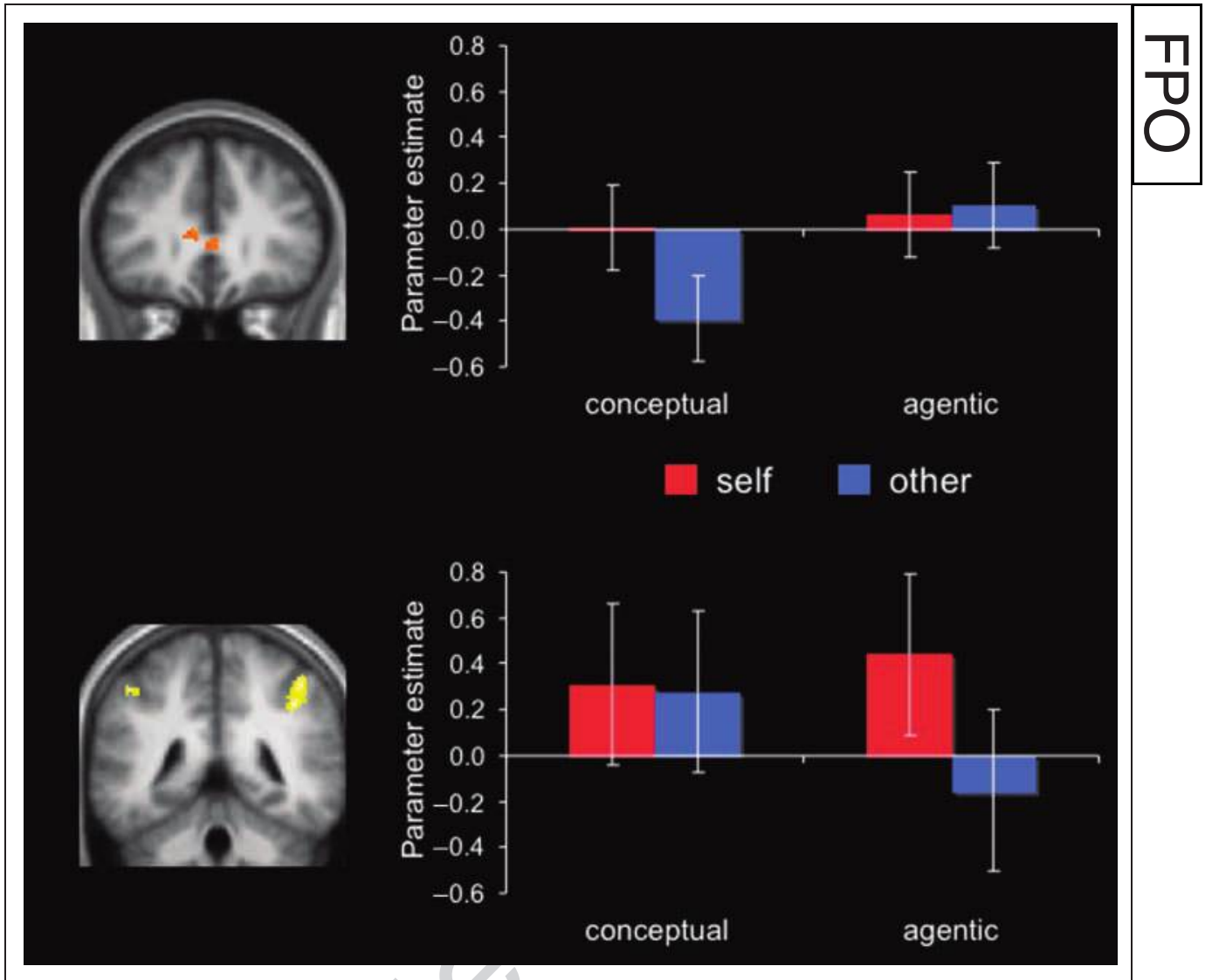

Table 4. Peak Voxel, Brodmann's Area, and Number of Voxels for Brain Regions Obtained from the Random-effects Contrast of Self and Other Trials on the Agentic Self-reference Task, $p<.05$, Corrected (Experiment 2)

\begin{tabular}{lrrrrrrr}
\hline Region & $x$ & $y$ & $z$ & BA & Voxels & $t$ \\
\hline Self $>$ Other & & & & & & \\
R Intraparietal sulcus & 44 & -32 & 44 & 40 & 1874 & 4.71 \\
L Intraparietal sulcus & -40 & -36 & 46 & 40 & 1631 & 4.83 \\
L Superior frontal gyrus & -22 & -4 & 54 & 6 & 1025 & 8.09 \\
L Middle frontal gyrus & -56 & 10 & 38 & 9 & 379 & 4.56 \\
R Superior frontal gyrus & 26 & 4 & 62 & 6 & 334 & 4.48 \\
Anterior cingulate cortex & 12 & 40 & 20 & 32 & 61 & 4.28
\end{tabular}

Other $>$ Self

\begin{tabular}{lrrrrrr} 
L Occipital & -14 & -96 & -10 & 17 & 231 & 4.36 \\
& -16 & -96 & 20 & 19 & 76 & 6.75 \\
R Occipital & 12 & -86 & 32 & 19 & 124 & 3.94 \\
L Fusiform & -28 & -54 & -10 & 19 & 60 & 6.28 \\
R Insula & 42 & -8 & -3 & 13 & 50 & 3.56 \\
Anterior cingulate cortex & -6 & 26 & 2 & 24 & 29 & 5.81 \\
\hline
\end{tabular}

Using an automated search algorithm (R. Poldrack, University of California Los Angeles) to define regions of interest around local maxima identified from this contrast (peak voxels separated by a minimum of $8 \mathrm{~mm}$ ), we examined whether activity in these areas was correlated with individuals' subsequent memory performance. For each participant, we segregated trials into those that were later remembered from those that were later forgotten as a function of whether items were agentically selected by participants or randomly selected by the computer (see Methods), resulting in four types of trials: self hits, self misses, other hits, and other misses. We then interrogated each of these regions using ANOVA procedures on the parameter estimates associated with each of these trial types to identify activity that was selectively correlated with subsequent memory performance for items agentically selected by the participants themselves, but not randomly selected by the computer. Specifically, such regions would be identified by three statistical features: (1) reliably greater BOLD response for self hits than self misses; (2) statistically indistinguishable response between other hits and other misses; and (3) a significant two-way interaction of Chooser (self, other) $\times$ Memory performance (hit, miss). Four such regions were identified: extensive posterior areas of the left and right IPS as well as more restricted regions in the left middle and right superior frontal gyri (Table 5). 
Table 5. Brain Regions Obtained from the Contrast of Self $>$ Other on the Agentic Self-reference Task (Experiment 2) that Also Demonstrated a Selective Correlation with Subsequent Memory for Self Trials

\begin{tabular}{|c|c|c|c|c|c|c|c|}
\hline Region & $x$ & $y$ & $z$ & Voxels & Self & Other & Interaction \\
\hline \multirow[t]{5}{*}{ R Intraparietal sulcus } & 16 & -66 & 50 & 382 & 0.016 & 0.767 & 0.012 \\
\hline & 30 & -56 & 54 & 354 & 0.009 & 0.698 & 0.013 \\
\hline & 34 & -74 & 42 & 286 & 0.029 & 0.974 & 0.010 \\
\hline & 40 & -64 & 56 & 207 & 0.005 & 0.487 & 0.010 \\
\hline & 34 & -64 & 30 & 159 & 0.012 & 0.569 & 0.004 \\
\hline L Intraparietal sulcus & -16 & -68 & 48 & 275 & 0.001 & 0.631 & 0.029 \\
\hline \multirow[t]{2}{*}{ L Middle frontal gyrus } & -48 & 32 & 32 & 62 & 0.003 & 0.971 & 0.014 \\
\hline & -40 & 32 & 22 & 51 & 0.003 & 0.904 & 0.013 \\
\hline R Superior frontal gyrus & 26 & 4 & 62 & 297 & 0.047 & 0.883 & 0.021 \\
\hline
\end{tabular}

The three rightmost columns report $p$ values associated with comparisons of hits and misses. The self and other columns report the significance level achieved by the $t$ tests of self hits $>$ self misses and other hits $>$ others misses, respectively. The interaction column reports the significance level achieved by the two-way Chooser (self, other) $\times$ Memory performance (hit, miss) interaction.

To confirm that memory performance on the agentic self-referential task was not likewise correlated with activity in $\mathrm{VMPFC}$, we defined a region of interest from separate functional runs in which participants completed a conceptual self-reference task identical to that used in Experiment 1 . Replicating earlier results, a region of vMPFC was identified from the contrast of self $>$ other (MNI coordinates: $0,46,0)$. However, analysis of the response in this region during the agentic self-reference task revealed no significant differences between hits and misses for either self-selected or computer-selected items (both $p s>.50$ ), demonstrating that VMPFC activity did not contribute to successful self-referential encoding for the agentic task.

Finally, we conducted an auxiliary random-effects, wholebrain analysis that identified regions demonstrating a significant two-way interaction of Target (self, other) $\times$ Task (agentic, conceptual). This analysis provided confirmatory support for the results described above. A region of vMPFC (772 voxels centered at 4, 46, 2) showed significantly greater difference for self $>$ other on the conceptual task than on the agentic task. Inversely, a region of the left IPS (325 voxels centered at $-40,-42,52$ ) showed significantly greater difference for self $>$ other on the agentic than conceptual task. At a relaxed statistical threshold of $p<.005$, a comparable region of the right IPS was also observed (97 voxels centered at $44,-32,48$ ). The equivalent analysis for Experiment 1 did not yield any brain regions, most likely due to its reduced statistical power (Figure 3).

\section{DISCUSSION}

Capitalizing on a well-characterized memory phenomenonthe self-reference effect-results of two experiments demonstrated distinct neural bases underlying different forms of self-referential processing. Replicating a number of earlier studies of conceptual self-reference, Experiment 1 demonstrated that making judgments of one's own personality traits (compared to those of another person) was associated with preferential modulation of vMPFC. However, activity in this region did not differentiate between self and other processing on a task that manipulated agentic aspects of self. Instead, self-involvement during the agentic choice task was associated with greater response in the IPS, a region previously linked to goal-directed action and motor planning. Experiment 2 extended these results by demonstrating that IPS activity during the initial agentic self-reference task was predictive of the mnemonic fate of items selected by participants. Words that went on to be remembered were associated with greater IPS response during the cardselection task than those that went on to be forgotten, but only if those words were "revealed" under cards actively selected by the participant (and not for those selected randomly by the computer). This correlation with subsequent memory suggests that, rather than being epiphenomenally "along for the ride," the IPS plays an active functional role in generating a sense of goal-directed authorship. In contrast, no such correlation between memory performance and neural response was observed in vMPFC, despite the fact that activity in this region has been shown previously to correlate with memory for items processed in reference to the conceptual self (Macrae et al., 2004).

Despite our subjective experience of being a coherent self, the current results suggest that some aspects of selfhood rely on distinct cognitive processes subserved by dissociable neural mechanisms. On the one hand, extant cognitive neuroscience findings have strongly linked MPFC to a variety of self-related processing in addition to knowledge of our own personality traits (i.e., conceptual self-reference). These include autobiographical memory (Maguire, 2001), monitoring one's own thoughts (Mason et al., 2007), reporting one's attitudes (Mitchell, 2009b), and the subjective experience of emotion (Phan et al., 2002), and have led to claims that "the self" engages a unique set of cognitive operations subserved specifically by MPFC (Schneider et al., 
Figure 3. Brain regions defined from the contrast of self $>$ other, separately for the conceptual (top) and agentic (bottom) self-reference tasks in Experiment 2. The right side of both panels displays parameter estimates associated with trials on the agentic task, segregated by whether words were subsequently remembered (solid bars) or forgotten (striped bars). Replicating Experiment 1, vMPFC differentiated between self and other trials on the conceptual task but did not distinguish between trial types on the agentic task. Furthermore, vMPFC activity did not correlate with later memory for self or other trials on the agentic task. In contrast, bilateral regions of the IPS showed greater activation for self $>$ other trials on the agentic self-reference task, and, moreover, activity in this region differentiated between items that were subsequently remembered (hits) versus forgotten (misses), but only for self trials on the agentic task. The bottom panel displays results from the right IPS

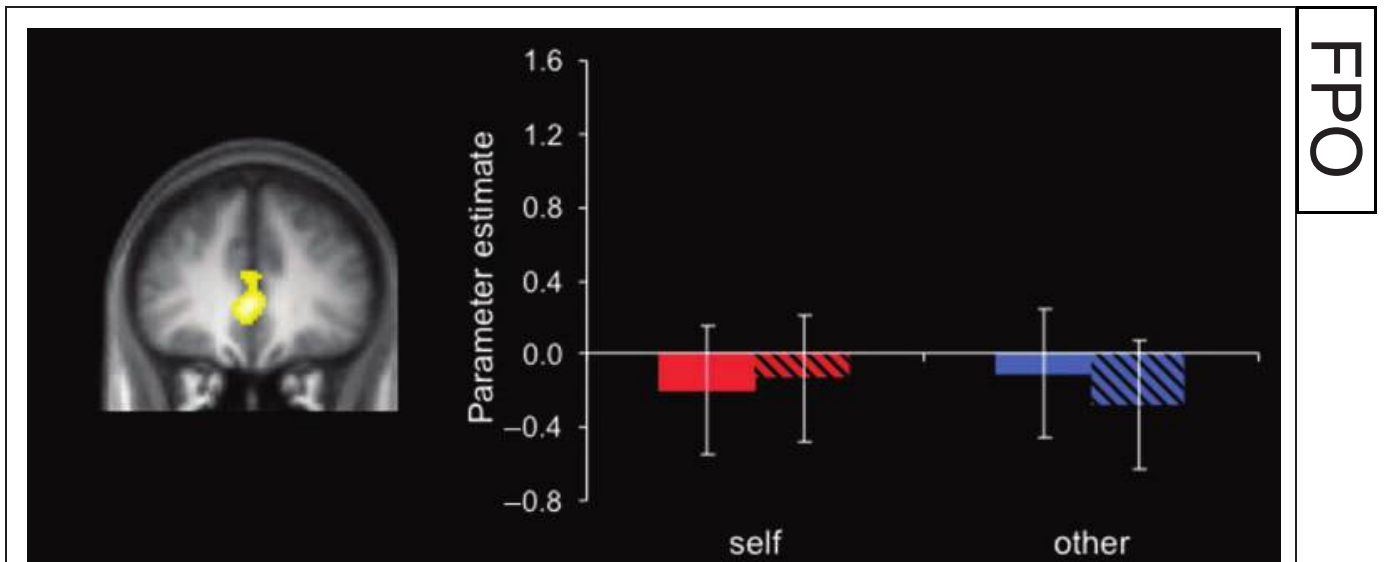

$\square$ hits $\mathbb{N}$ misses

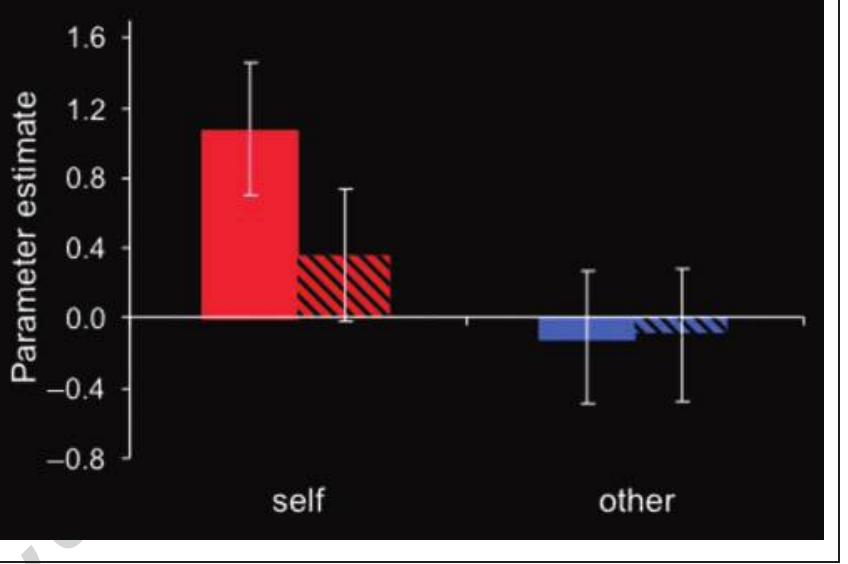

2008; Uddin et al., 2007; Northoff \& Bermpohl, 2004; cf. Gillihan \& Farah, 2005). In contrast, the current study demonstrates that one central aspect of selfhood-the sense of being an active, voluntary, and free agent of change in the environment-does not rely on cortical midline structures such as MPFC. This pattern of results is consistent with the recently articulated hypothesis that MPFC subserves abstract, probabilistic, and "fuzzy" cognitive processes, such as those inherent in conceptual self-reference, the experience of emotion, and introspective awareness (Mitchell, 2009b). In contrast, MPFC contributes less to concrete, discrete, and specific mental operations of the kind needed to select a particular course of action and monitor its specific outcome in the environment. In the case of agentic selfprocessing as operationalized here, such discrete and specific processing appears instead to be subserved by the IPS.

Although the conceptual and agentic tasks clearly differed from each other in a number of ways, each was designed to isolate a discrete aspect of selfhood. As operationalized in dozens of studies since the 1970s (Symons \& Johnson, 1997), the conceptual self-reference task manipulated whether perceivers introspected about their own personality traits and dispositions or judged those of a familiar, but not personally known, other. In contrast, following earlier work (Cloutier \& Macrae, 2008), during the agentic selfreference task, participants either engaged in goal-directed action toward an idiosyncratically chosen item or watched passively as an item was selected randomly. The central question of interest in the current studies was whether these two ways of manipulating self-involvement (conceptual vs. agentic) rely on overlapping or dissociated neural substrates. Although differences between the two paradigms undermine any direct comparison between the conceptual and agentic tasks, the logic of the analyses used in both experiments obviates the need to contrast the tasks to each other. Global differences between the tasks, such as obvious disparities in the visual display or task instructions, were subtracted out by contrasting the self and other conditions from within the same task. As such, comparisons of interest focused on whether the process(es) isolated by the agentic task draws on the same underlying neural substrates as those isolated by the conceptual task. The observation of a three-way interaction of Task (conceptual, agentic) $\times$ Target (self, other) $\times$ Brain region $($ vMPFC, IPS) addressed this question without the need to compare the tasks to each directly, suggesting that the aspects of self isolated by the agentic task do not overlap completely with those aspects of self isolated by the conceptual task.

Likewise, the subsequent memory approach used in Experiment 2 further underscores the specificity of the cognitive processes isolated by these tasks. Such analyses have been used frequently in the memory literature to 
distinguish brain regions that make significant processing contributions to task performance from those that are $\mathrm{CO}-$ activated by the task for extraneous or peripheral reasons (Paller \& Wagner, 2002; Wagner, Koutstaal, \& Schacter, 1999). For example, although motor cortex might be engaged by an orienting task that requires a behavioral response, activity in this region would be unlikely to predict whether an item goes on to be remembered, because motor processing rarely contributes importantly to successful memory encoding. The current data capitalize on this aspect of the subsequent memory approach in two ways. First, our findings underscore the insensitivity of vMPFC to differences between agentic self and other. In addition to failing to distinguish self from other during the agentic task, vMPFC activity also failed to differentiate between remembered and forgotten items on the agentic task (regardless of chooser), suggesting that any vMPFC coactivity during the agentic task was not functionally important to task performance. More importantly, IPS activity was predictive of later memory for items actively chosen by the self, but not for "other" items. This result suggests that, rather than being modulated by the agentic task generally, IPS activity contributes selectively to memory for one's own actions. Taken together, these results suggest that the two self-referential tasks isolate distinct cognitive processes with distinct neural bases.

\section{Functional Basis of the "Agentic" Self}

The link between representations of one's actions and the IPS has been presaged by a number of researchers. For example, in a series of studies, Grafton, Hamilton, and colleagues have demonstrated the role of the anterior IPS in maintaining a representation of the target of one's action and planning the motor commands needed to bring about an intended result (Grafton \& Hamilton, 2007; Tunik, Rice, Hamilton, \& Grafton, 2007; Hamilton \& Grafton, 2006). For example, Hamilton and Grafton (2006) demonstrated that regions of the IPS were activated during the attribution of a particular goal by showing that these regions adapted to repeated actions directed at the same goal, responding less to a second reach for the same object than during the first one. Likewise, a number of other studies have found that regions in the IPS play an important role in working memory for both visuospatial and kinesthetic information related to one's own actions (Fiehler, Burke, Engel, Bien, \& Rosler, 2008; Goodale \& Milner, 1992). For example, Fiehler et al. (2008) showed that left IPS activity increased linearly as participants were obliged to maintain active representations of increasing numbers of recent actions. Together, these results suggest that the IPS may play an important role in representing one's goals and the means by which to realize them, and may therefore be engaged preferentially by situations in which one freely authors an action (i.e., in which the agentic self is engaged).

This interpretation is consistent with previous work by Cloutier and Macrae (2008), who found that active, free choice was critical to producing better memory for selected items. When participants were denied the opportunity to choose a card by instead simply having one assigned to them, memory for such items was equivalent to those selected by another person. Future work will be needed to address whether preventing subjects from engaging in active, free choice would likewise reduce activation in the region of the IPS associated here with agentic selfreference. Although both the selection and execution of an action were not disentangled in the current study, we suspect that the IPS will contribute most prominently to situations in which participants actively choose a particular action from among equally possible alternatives.

Intriguingly, in addition to contributing to representations of one's own goal-directed actions, the IPS has been implicated in perceiving others' actions. A sizeable number of neuroimaging studies have characterized a "mirror neuron system" in the human brain (e.g., Buccino et al., 2001, 2004; Iacoboni et al., 1999). Such work has found that, although the most robust activity in the IPS accompanies execution of an action, there is also a measurable response in this region when participants merely observe someone else acting. However, in the current study, no modulation of IPS activity over baseline was observed during "other" trials on the agentic task. This observation is consistent with mirror neuron accounts, which suggest that regions implicated in mirroring (such as the IPS) will be engaged only when an individual has direct perceptual input (either visual or auditory) about the current goal-directed movements of another person. Because our participants did not directly perceive the movements of another person but were simply informed about the outcome of a choice that was not theirs, we strongly suspect that the IPS should not be modulated by the "other" condition of the agentic self task. This hypothesis is indirectly supported by the observation that IPS activity was functionally related to behavior (i.e., memory performance) only for agentically chosen self trials, but not passively viewed other trials.

Likewise, the specificity of the correlations between IPS activity and subsequent memory rules out an important class of alternative explanations for these results. Although one might expect participants to devote greater attention to trials on which they select a card themselves (than those on which a card is randomly selected), the fact that the subsequent memory effect in the IPS was observed only for self-selected items makes it unlikely that activity in this region indexed global differences in attention between the two conditions. If IPS activity reflected greater attention to self items, and if such enhanced attention supports better memory encoding for those items, then IPS activity would have also distinguished those other-selected items to which participants paid close attention, and thus, later remembered from those to which participants attended superficially and therefore forgot. However, the selective relation between IPS activity and memory only for self items suggests that this region contributes specifically to cognitive processes important for representing the targets 
of one's own actions. Moreover, that this subsequent memory effect was not observed in brain areas typically implicated in deeper levels of encoding-such as inferior frontal and medial temporal regions (Otten, Henson, \& Rugg, 2001)—suggests that the current operationalization of agentic self-reference did not simply produce more elaborative, "deeper" encoding of chosen information.

\section{Conclusion}

Although the current results suggest the separability of two components of the self, they do not address the question of whether these two components are instantiated by cognitive mechanisms dedicated exclusively to self-processing. In a recent review, Gillihan and Farah (2005) pointed out that many cognitive mechanisms seem to be involved in self-referential processing, not all of which may be devoted solely to the self. Nevertheless, the current research suggests that different aspects of the self rely on distinct neural systems and, moreover, that these different systems can each independently support common effects of selfinvolvement such as enhanced memory for items relevant to the self. Although we have focused on two particular components of our self representations - the conceptual and agentic selves - other commentators have discussed a number of other potential candidates for dissociable aspects of the self. For example, in one influential treatment, Neisser (1988) described a number of distinct components of selfhood, such as a "conceptual self" that represents our understanding of our own personality traits and dispositions; an "ecological self" that represents our sense of authoring our own actions in the environment (similar to what we have here called the "agentic self"); a "narrative self" that maintains our sense of personal history and autobiographical memory; and a "social self" that reflects an understanding of our roles, obligations, and rights as individuals with a specific place in our social networks (also see Boyer et al., 2005; Gallagher, 2000). These proposed aspects of the self are fertile ground for future research, including investigations aimed at characterizing dissociable cognitive mechanisms that support these forms of self-processing. We hope that future work will elucidate how these multiple aspects of selfhood are bound together into the coherent sense of a unitary self that marks healthy human cognition.

\section{Acknowledgments}

We thank D. L. Ames and A. Jenkins for their advice and assistance. This work was supported by the National Science Foundation (BCS 0642448). Data were collected at the Athinoula A. Martinos Center for Biomedical Imaging, which is supported by the National Center for Research Resources (P41RR14075) and the MIND Institute.

Reprint requests should be sent to Prof. Jason P. Mitchell, Department of Psychology, Harvard University, Northwest Science Building 220.01, 52 Oxford Street, Cambridge, MA 02138, or via e-mail: jason_mitchell@harvard.edu.

\section{REFERENCES}

Ames, D. L., Jenkins, A. C., Banaji, M. R., \& Mitchell, J. P. (2008). Taking another's perspective increases self-referential neural processing. Psychological Science, 19, 642-644.

Bowers, G. H., \& Gilligan, S. G. (1979). Remembering information related to one's self. Journal of Research in Personality, 13, 420-432.

Boyer, P., Robbins, P., \& Jack, A. I. (2005). Varieties of self-systems worth having. Consciousness and Cognition. 14, 647-660.

Brewer, J. B., Zhao, Z., Desmond, J. E., Glover, G. H., \& Gabrieli, J. D. (1998). Making memories: Brain activity that predicts how well visual experience will be remembered. Science, 281, 1185-1187.

Buccino, G., Binkofski, F., Fink, G. R., Fadiga, L., Fogassi, L., Gallese, V., et al. (2001). Action observation activates premotor and parietal areas in a somatotopic manner: An fMRI study. European Iournal of Neuroscience, 13, 400-404.

Buccino, G., Vogt, S., Ritzl, A., Fink, G. R., Zilles, K., Freund, H. J., et al. (2004). Neural circuits underlying imitation learning of hand actions: An event-related fMRI study. Neuron, 42, 323-334.

Buckner, R. L., \& Carroll, D. C. (2007). Self-projection and the brain. Trends in Cognitive Sciences, 11, 49-57.

Cloutier, J., \& Macrae, C. N. (2008). The feeling of choosing: Self-involvement and the cognitive status of things past. Consciousness and Cognition, 17, 125-135.

Dale, A. M. (1999). Optimal experimental design for event-related fMRI. Human Brain Mapping, 8, 109-114.

D’Argembeau, A., Ruby, P., Collette, F., Degueldre, C., Balteau, E., Luxen, A., et al. (2007). Distinct regions of the medial prefrontal cortex are associated with self-referential processing and perspective taking. Journal of Cognitive Neuroscience, 19, 935-944.

Davachi, L., Mitchell, J. P., \& Wagner, A. D. (2003). Multiple routes to memory: Distinct medial temporal lobe processes build item and source memories. Proceedings of the National Academy of Sciences. U.S.A., 100, 2157-2162.

Doody, R. S., \& Jankovic, J. (1992). The alien hand and related signs. Journal of Neurologv. Neurosurgerv and Psychiatry 55, 806-810.

Feinberg, T. E., Schindler, R. J., Flanagan, N. G., \& Haber, L. D. (1992). Two alien hand syndromes. Neurology, 42, 19-24.

Fiehler, K., Burke, M., Engel, A., Bien, S., \& Rosler, F. (2008). Kinesthetic working memory and action control within the dorsal stream. Cerebral Cortex, 18, 243-253.

Frith, C. (2005). The self in action: Lessons from delusions of control. Consciousness and Cognition, 14, 752-770.

Gallagher, S. (2000). Philosophical conceptions of the self: Implications for cognitive science. Trends in Cognitive Sciences, 4, 14-21.

Gillihan, S. J., \& Farah, M. J. (2005). Is self special? A critical review of evidence from experimental psychology and cognitive neuroscience. Psychological Bulletin, 131, 76-97.

Goodale, M. A., \& Milner, A. D. (1992). Separate visual pathways for perception and action. Trends in Neurosciences, 15, 20-25.

Grafton, S. T., \& Hamilton, A. F. (2007). Evidence for a distributed hierarchy of action representation in the brain. Human Movement Science, 26, 590-616.

Hamilton, A. F., \& Grafton, S. T. (2006). Goal representation in human anterior intraparietal sulcus. Dournal of Neuroscience, 26, 1133-1137. 
Heatherton, T. F., Wyland, C. L., Macrae, C. N., Demos, K. E., Denny, B. T., \& Kelley, W. M. (2006). Medial prefrontal activity differentiates self from close others. Social Cognitive and Affective Neuroscience, 1, 18-25.

Humphrey, N. (2000). One self: A meditation on the unity of consciousness. Social Research, 67, 32-39.

Iacoboni, M., Woods, R. P., Brass, M., Bekkering, H., Mazziotta, J. C., \& Rizzolatti, G. (1999). Cortical mechanisms of human imitation. Science, 286, 2526-2528.

James, W. (1890). Principles of psychology. New York: Holt, Rinehart \& Wilson.

Jenkins, A. C., Macrae, C. N., \& Mitchell, J. P. (2008). Repetition suppression of ventromedial prefrontal activity during judgments of self and others. Proceedings of the National Academy of Sciences, U.S.A., 105, 4507-4512.

Johnson, S. C., Baxter, L. C., Wilder, L. S., Pipe, J. G., Heiserman, J. E., \& Prigatano, G. P. (2002). Neural correlates of self-reflection. Brain, 125, 1808-1814.

Jones, E. E., \& Nisbett, R. E. (1971). The actor and observer: Divergent perceptions of the causes of behavior. Morristown, NJ: General Learning Press.

Kahneman, D., Knetsch, J. L., \& Thaler, R. H. (1990). Experimental tests of the endowment effect and the Coase theorem. Journal of Political Economy, 98, 1325-1348.

Kelley, W. M., Macrae, C. N., Wyland, C. L., Caglar, S., Inati, S., \& Heatherton, T. F. (2002). Finding the self? An event-related fMRI study. Journal of Cognitive Neuroscience, 14, 785-794.

Kelley, W. M., Miezin, F. M., McDermott, K. B., Buckner, R. L., Raichle, M. E., Cohen, N. J., et al. (1998). Hemispheric specialization in human dorsal frontal cortex and medial temporal lobe for verbal and nonverbal memory encoding. Neuron, 20, 927-936.

Klein, S. B., Loftus, J., \& Kihlstrom, J. F. (1996). Self-knowledge of an amnesic patient: Toward a neuropsychology of personality and social psychology. Journal of Experimental Psychology: General, 125, 250-260.

Klein, S. B., Rozendal, K., \& Cosmides, L. (2002). A social-cognitive neuroscience analysis of the self. Social Cognition, 20, 105-135.

Langer, E. J. (1975). The illusion of control. Journal of Personality and Social Psychology, 32, 311-328.

Langer, E. J. (1977). The psychology of chance. Journal for the Theory of Social Behaviour, 7, 185-203.

Loftus, G. R., \& Masson, M. E. J. (1994). Using confidence intervals in within-subject designs. Psychonomic Bulletin E Review, 1, 476-490.

Lou, H. C., Luber, B., Crupain, M., Keenan, J. P., Nowak, M., Kjaer, T. W., et al. (2004). Parietal cortex and representation of the mental self. Proceedings of the National Academy of Sciences, U.S.A., 101, 6827-6832.

Macrae, C. N., Moran, J. M., Heatherton, T. F., Banfield, J. F., \& Kelley, W. M. (2004). Medial prefrontal activity predicts memory for self. Cerebral Cortex, 14, 647-654.

Maguire, E. A. (2001). Neuroimaging studies of autobiographical event memory. Philosophical Transactions of the Royal Society of London, Series B, Biological Sciences, 356, 1441-1451.

Markus, H. (1977). Self-schemata and processing information about the self. Journal of Personality and Social Psychology, 35, 63-78.

Mason, M. F., Norton, M. I., Van Horn, J. D., Wegner, D. M., Grafton, S. T., \& Macrae, C. N. (2007). Wandering minds: The default network and stimulus-independent thought. Science, 315, 393-395.

Mitchell, J. P. (2009a). Inferences about other minds. Philosophical Transactions of the Royal Society of London, Series B, Biological Sciences, 364, 1309-1316.
Mitchell, J. P. (2009b). Social psychology as a natural kind. Trends in Cognitive Sciences, 13, 246-251.

Mitchell, J. P., Macrae, C. N., \& Banaji, M. R. (2006). Dissociable medial prefrontal contributions to judgments of similar and dissimilar others. Neuron, 50, 655-663.

Moran, J. M., Macrae, C. N., Heatherton, T. F., Wyland, C. L., \& Kelley, W. M. (2006). Neuroanatomical evidence for distinct cognitive and affective components of self. Journal of Cognitive Neuroscience, 18, 1586-1594.

Neisser, U. (1988). Five kinds of self-knowledge. Philosophical Psychology, 1, 35-59.

Northoff, G., \& Bermpohl, F. (2004). Cortical midline structures and the self. Trends in Cognitive Sciences, 8, 102-107.

Otten, L. J., Henson, R. N. A., \& Rugg, M. D. (2001). Depth of processing effects on neural correlates of memory encoding: Relationship between finding from across- and within-task comparisons. Brain, 124, 399-412.

Paller, K. A., \& Wagner, A. D. (2002). Observing the transformation of experience into memory. Trends in Cognitive Sciences, 6, 93-102.

Phan, K. L., Wager, T., Taylor, S. F., \& Liberzon, I. (2002). Functional neuroanatomy of emotion: A meta-analysis of emotion activation studies in PET and fMRI. Neuroimage, 16, 331-348.

Rogers, T. B., Kuiper, N. A., \& Kirker, W. S. (1977). Self-reference and the encoding of personal information. Journal of Personality \& Social Psychology, 35, 677-688.

Schmitz, T. W., Kawahara-Baccus, T. N., \& Johnson, S. C. (2004). Metacognitive evaluation, self-relevance, and the right prefrontal cortex. Neuroimage, 22, 941-947.

Schneider, F., Bermpohl, F., Heinzel, A., Rotte, M., Walter, M., Tempelmann, C., et al. (2008). The resting brain and our self: Self-relatedness modulates resting state neural activity in cortical midline structures. Neuroscience, 157, 120-131.

Stirling, J. D., Hellewell, J. S., \& Quraishi, N. (1998).

Self-monitoring dysfunction and the schizophrenic symptoms of alien control. Psychological Medicine, 28, 675-683.

Symons, C. S., \& Johnson, B. T. (1997). The self-reference effect in memory: A meta-analysis. Psychological Bulletin, 121, 371-394.

Tunik, E., Rice, N. J., Hamilton, A., \& Grafton, S. T. (2007). Beyond grasping: Representation of action in human anterior intraparietal sulcus. Neuroimage, 36(Suppl. 2), T77-T86.

Uddin, L. Q., Iacoboni, M., Lange, C., \& Keenan, J. P. (2007). The self and social cognition: The role of cortical midline structures and mirror neurons. Trends in Cognitive Sciences, 11, 153-157.

Wagner, A. D., Koutstaal, W., \& Schacter, D. L. (1999). When encoding yields remembering: Insights from event-related neuroimaging. Philosophical Transactions of the Royal Society of London, Series B, Biological Sciences, 354, 1307-1324.

Wagner, A. D., Schacter, D. L., Rotte, M., Koutstaal, W., Maril, A., Dale, A. M., et al. (1998). Building memories: Remembering and forgetting of verbal experience as predicted by brain activity. Science, 281, 1188-1191.

Wegner, D. M. (2002). The illusion of conscious will. Cambridge, MA: MIT Press.

Zhu, Y., Zhang, L., Fan, J., \& Han, S. (2007). Neural basis of cultural influence on self-representation. Neuroimage, 34, $1310-1316$

Zysset, S., Huber, O., Ferstl, E., \& von Cramon, D. Y. (2002). The anterior frontomedian cortex and evaluative judgment: An fMRI study. Neuroimage, 15, 983-991. 